\title{
Microvariability of the blazar OJ $287^{\star}$
}

\author{
G.B. Jia, X.F. Cen, H.Y. Ma, and J.C. Wang \\ Yunnan Observatory, Academia Sinica, P.O. Box 110, Kunming, Yunnan, P.R. China
}

Received February 2, 1996; accepted September 19, 1997

\begin{abstract}
Results of $B V R I$ CCD photometric monitoring and fast photometry in the $I$ band for the blazar OJ 287 in 1994-1995 are presented. The predicted outburst of the blazar had been observed, the maximum appeared in 1994 November, after that, the luminosity decreased by $1.6 \mathrm{mag}$ for about three months and dropped to near the level in 1994 April. Rapid variations with timescales of minutes, hours and one day were observed in our observations, with small amplitude. The amplitude of the most rapid variations (minutes) is in the range of $0.043-0 .{ }^{\mathrm{m}} 12$. The results of the statistical analysis of the fast photometry data are as follows. In five nights out of six rapid variability appeared in the sampling interval, which ranged from 3.4 minutes to 6.5 minutes, with a rms amplitude of flux variations of $1.70 \%-2.3 \%$.

The rapid variability can be explained by the model of shocks within turbulent jet or of vortices and magnetic flux tubes on accretion disk.
\end{abstract}

Key words: BL Lacertae objects: individual: OJ 287 - galaxies: active

\section{Introduction}

OJ 287 has all the characteristics of blazars, and it is one of the sources studied the most extensively. Variability studies of the blazar concentrated mostly on the frequencies lower than $10^{15} \mathrm{~Hz}$, due to its weak emission at high frequencies. In the UV range, Maraschi et al. (1986) reported variations on a timescale of two days. In the optical range, flux variations of some periodicities were reported, with periods ranging from 13 minutes to 11.65 years (e.g. Visvanathan \& Elliot 1973; Frohlich et al. 1974; Kinman 1974; Carrasco et al. 1985; Valtaoja et al. 1985; Xie et al. 1987; Sillanpää et al. 1988; Kidger et al. 1992; Turner et al. 1994). Long-term variations reach 4 mag in the $B$ band (Webb et al. 1988). The question of the existence of

\footnotetext{
Send offprint requests to: G.B. Jia

* Table 5 is only available at the CDS via anonymous ftp 130.79.128.5 or http://cdsweb.u-strasbg.fr/Abstract.html
}

microvariations (lasting a few minutes to hours) in the optical flux of blazars, until recently, remained unanswered. They are either discrete events or parts of long term (night to night) variations (Carini et al. 1992). Intraday variations in optical flux were reported by some authors (e.g. Epstein et al. 1972; Visvanathan \& Elliot 1973; Frohlich et al. 1974; McGimsey et al. 1975; Véron \& Véron 1975; Carrasco et al. 1985; Valtaoja et al. 1985; Miller et al. 1989; Carini et al. 1992; Sillanpää et al. 1992; Benitez et al. 1994; Sillanpää et al. 1994; Takalo et al. 1994; Kidger et al. 1995). The polarization level in the optical region for OJ 287 ranged from $0.4 \%$ to $43 \%$ (Hagen-Thorn 1980), the position angle varied on timescales from years to hours (Takalo et al. 1994 and references therein). The polarization and position angle are frequency-dependent (Valtaoja et al. 1991 and references therein), the FDP and the FDPA were variable with timescales from day to hours, and both the strength and/or sign of the FDP or FDPA can also vary (Takalo et al. 1994 and references therein).

Microvariability of OJ 287 in the infrared was reported (e.g. Epstein et al. 1972; Wolstencroft et al. 1982; Holmes et al. 1984; Lorenzetti et al. 1989; Takalo et al. 1992; Kidger et al. 1994). At radio frequencies, intraday variability was also reported (e.g. Epstein et al. 1972; Valtaoja et al. 1985; Dreher et al. 1986; De Bruyn 1988; Kinzel et al. 1988), and there is a strong correlation between the variabilities in the optical and in radio with short (intraday) or long lags (Wagner \& Witzel 1995 and references therein).

Since 1987, we have monitored OJ 287 and searched for rapid variability. However in 1990-1992 only variability with a timescale of one day was observed (Guibin et al. 1995). A large outburst during 1994 for OJ 287 was predicted by Sillanpää et al. (1988) based on an average 11.65 years interval between major outbursts. Sillanpää et al. (1996) claimed that the outburst had occurred in 1994 and the peak of the outburst was observed around November 10. In 1994-1995, we continued to search for the rapid variability and observed variability with timescales of minutes, hours and one day in OJ 287. 


\section{Observations}

In 1994-1995, BVRI photometric monitoring and fast photometry at $I$ band for OJ 287 were carried out with the CCD attached to the focal reducer of the 1-m RCC telescope at the Yunnan Observatory, China. The CCD (Tek $512 \mathrm{mc}$ ) has $512 \times 512$ pixels of $27 \times 27 \mu \mathrm{m}^{2}$. The field of view is $7.4^{\prime} \times 7.4^{\prime}$. The characteristics of the filter are the same as that described in Schild (1983). The comparison star sequence is taken from Smith et al. (1985). The journal of observations is given in Table 1. The integration time and individual measurement errors in the $I$ magnitude for the object and comparison stars 4, 10 and 11 as well as for a fainter star 5 (Visvanathan \& Elliot 1973) are tabulated in Table 2 . The averages of $\mathrm{N} / \mathrm{S}$ ratio of flux for the object and stars 4 and 10 in each night are respectively listed in lines 2, 3 and 4 in Table 4 .

Table 1. Fast photometry mode: journal of observations at $I$ band

\begin{tabular}{cccc}
\hline \hline Date & $\begin{array}{c}\text { Duration of } \\
\text { Observations(hr.) }\end{array}$ & $\begin{array}{c}\text { Number of } \\
\text { observations }\end{array}$ & $\begin{array}{c}\text { Average sampling } \\
\text { interval (min.) }\end{array}$ \\
\hline $4 / 11 / 94$ & 0.60 & 8 & 4.50 \\
$11 / 7 / 94$ & 0.92 & 14 & 3.93 \\
$11 / 8 / 94$ & 1.01 & 18 & 3.35 \\
$2 / 8 / 95$ & 3.27 & 30 & 6.53 \\
$2 / 22 / 95$ & 2.74 & 30 & 5.50 \\
$2 / 23 / 95$ & 2.65 & 38 & 4.17 \\
\hline \hline
\end{tabular}

\section{Data reduction}

The method of data reduction had been detailed in the previous paper (Guibin et al. 1995). The comparison stars 4, 10 and 11 are intercalibrated in the $I$ band each night. The magnitudes outside the atmosphere of the intercalibrated comparison stars are slightly different from the values given by Smith et al. (1985) in some nights. The differences reach about $0.01,0 \cdot 01-0.02$ and $0.01-0.03$ for star 4, 10 and 11 respectively. The uncertainties in the magnitudes of intercalibrated comparison stars are $0{ }^{\mathrm{m}} 006-0{ }^{\mathrm{m}} 02,0.01-0 .{ }^{\mathrm{m}} 03$ and $00^{\mathrm{m}} 01-0{ }^{\mathrm{m}} 02$ for star 4, 10 and 11 respectively. In the $B V R$ bands, the magnitudes and errors given by Smith et al. (1995) are used. The weighted average of the extinction values measured on stars 4, 10 and 11 is used to calibrate the object. The calibrated magnitudes of OJ 287 are tabulated in Table 5 (available electronically). The uncertainties in the table include the errors on the measured magnitudes of the object and the comparison stars and the uncertainties in the magnitudes of the comparison stars. The colour index was calculated from two consecutive observations in different bands. The average of colour indices and magnitudes in $B V R I$ are listed in Table 3 for each night.

In order to search for rapid variability, the measured magnitude of the blazar, star 4 and star 10 are directly compared, the differential magnitudes Dm1 between the blazar and star 4 and Dm2 between star 10 and star 4, are shown in Figs. 1-6, The uncertainty of each data point in the figures is calculated on the basis of the errors in the measurement of the differential magnitude of the two mutually-compared stars. Comparing the Dm1 and Dm2 light curves one can determine whether the blazar is variable or not. Also, we calculate the flux ratio based on the differential magnitude, $R_{1}$ and $R_{2}$, being the flux ratios of OJ 287 to star 4 and of star 10 to star 4 respectively. The flux ratio has the same variance terms as the differential magnitude. The averages of relative errors of $R_{1}$ and $R_{2}$ and the rms values of $R_{1}$ and $R_{2}$ relative to its average are also calculated. The rms value could represent a measure of the amplitude of variations of flux ratio during the sampling interval in the night. We compare the proportion of the rms value to the average of the flux ratio with the mean of relative errors of flux ratio. If the former is definitely greater than the later, the rapid variability of the flux ratio during the sampling interval is observed; if the former is about the same as the latter, the flux ratio is not variable, it means that the $\sigma^{2}$ (var) are all equal to zero in the two objects. If $R_{1}$ is variable and $R_{2}$ has no variability, the conclusion of variations of the blazar during sampling interval can be obtained. Furthermore, the $\chi^{2}$ (relative to the weighted mean of the data) test was carried out, thus the significance of variability can be seen.

\section{Results}

\subsection{BVRI monitoring}

One can see from Table 3 that the blazar was brightening during 1994; on 1994 November 8 the luminosity reached a maximum $I=13.178 \mathrm{mag}$ and increased by $1.6 \mathrm{mag}$ as compared with that on 1994 April 11. Afterward, the luminosity declined and until 1995 February dropped down to near the 1994 April level. This agrees with the results reported by Sillanpää et al. (1996). The variability on a timescale of one day in $I$ band was only 0.04 mag. In other bands the error is too large to determine if there is variability.

\subsection{Fast photometry in the I band}

One can see the following results from Table 5 (available electronically) and Figs. 1-6.

1. On 1994 April 11 (Fig. 1) we observed a change of magnitude of $0{ }^{\mathrm{m}} 06 \pm 0{ }^{\mathrm{m}} 015$ in about 8 minutes. 2. On 1994 November 7 (Fig.2) the light curve apparently began to fall at $.394 \mathrm{~d}$, reached a minimum at $.401 \mathrm{~d}$, then rised up. If this is real, the blazar has decreased by approximately $0.12 \pm 0.014 \mathrm{mag}$ in ten minutes and brightened about $0.07 \pm 0.014 \mathrm{mag}$ in about 3 minutes. 3. On 1994 November 8 (Fig. 3) the light curve began to fall at .387d, reached a minimum at $.393 \mathrm{~d}$, then rised up. If the variations are real, the magnitude has increased by $0.09 \pm 0.013$ in an interval of about 13 minutes and decreased by $0.09 \pm 0.014$ 
Table 2. Integrated time and individual measurement error on the $I$ magnitudes of OJ 287 and comparison stars

\begin{tabular}{|cc|ccccc|}
\hline \hline Date & $\begin{array}{c}\text { integrated } \\
\text { time(sec.) }\end{array}$ & OJ 287 & \multicolumn{5}{c|}{ Error of individual measurement (mag) } \\
& 150 & $0.011-0.013$ & 0.005 & 0.007 & $0.008-0.010$ & $0.014-0.016$ \\
$4 / 11 / 94$ & 100 & $0.006-0.007$ & $0.006-0.007$ & $0.008-0.011$ & $0.010-0.012$ & $0.017-0.021$ \\
$11 / 7 / 94$ & 100 & $0.006-0.007$ & $0.006-0.007$ & $0.009-0.010$ & $0.010-0.011$ & $0.017-0.019$ \\
$11 / 8 / 94$ & 200 & $0.008-0.010$ & $0.004-0.005$ & $0.006-0.007$ & $0.007-0.008$ & $0.010-0.011$ \\
$2 / 8 / 95$ & 200 & $0.007-0.010$ & $0.004-0.005$ & $0.005-0.007$ & $0.006-0.008$ & $0.011-0.012$ \\
$2 / 22 / 95$ & 150 & $0.011-0.012$ & 0.005 & $0.007-0.008$ & $0.008-0.009$ & $0.013-0.015$ \\
$2 / 23 / 95$ & \multicolumn{6}{l}{} \\
\hline \hline
\end{tabular}

Table 3. The average magnitude and colour index of OJ 287

\begin{tabular}{lccccccc}
\hline \hline UT Date & $\langle B\rangle$ & $\langle V\rangle$ & $\langle R\rangle$ & $\langle I\rangle$ & $\langle B-V\rangle$ & $\langle V-R\rangle$ & $\langle R-I\rangle$ \\
\hline $4 / 11 / 94$ & & & & 14.775 & & & \\
& & & & $(0.005)$ & & 0.21 & 0.54 \\
$11 / 7 / 94$ & & 13.90 & 13.69 & 13.178 & & $(0.05)$ & $(0.04)$ \\
& & $(0.03)$ & $(0.04)$ & $(0.003)$ & & 0.36 & 0.50 \\
$11 / 8 / 94$ & 14.50 & 14.09 & 13.73 & 13.217 & 0.41 & $(0.05)$ & $(0.04)$ \\
& $(0.05)$ & $(0.03)$ & $(0.04)$ & $(0.002)$ & $(0.06)$ & 0.42 & 0.65 \\
$2 / 8 / 95$ & 16.05 & 15.57 & 15.15 & 14.550 & 0.48 & $(0.05)$ & $(0.04)$ \\
& $(0.05)$ & $(0.03)$ & $(0.04)$ & $(0.002)$ & $(0.06)$ & 0.31 & 0.54 \\
$2 / 22 / 95$ & 16.11 & 15.69 & 15.30 & 14.728 & 0.50 & $(0.06)$ & $(0.04)$ \\
& $(0.07)$ & $(0.02)$ & $(0.04)$ & $(0.002)$ & $(0.08)$ & 0.39 & 0.57 \\
$2 / 23 / 95$ & 16.23 & 15.71 & 15.32 & 14.724 & 0.52 & $(0.06)$ & $(0.04)$ \\
& $(.08)$ & $(0.04)$ & $(0.04)$ & $(0.002)$ & $(0.09)$ & & \\
\hline \hline
\end{tabular}

Table 4. The results of statistical analysis of fast photometry

\begin{tabular}{ccccccc}
\hline \hline Date & $4 / 11 / 94$ & $11 / 7 / 94$ & $11 / 8 / 94$ & $2 / 8 / 95$ & $2 / 22 / 95$ & $2 / 23 / 95$ \\
$\left\langle\frac{\sigma}{F}\right\rangle_{s}$ & $1.0810^{-2}$ & $6.3810^{-3}$ & $5.5810^{-3}$ & $7.9510^{-3}$ & $8.6610^{-3}$ & $1.0210^{-2}$ \\
$\left\langle\frac{\sigma}{F}\right\rangle_{4}$ & $4.6110^{-3}$ & $6.3810^{-3}$ & $5.5810^{-3}$ & $4.3010^{-3}$ & $3.7210^{-3}$ & $4.6110^{-3}$ \\
$\left\langle\frac{\sigma}{F}\right\rangle_{10}$ & $6.4510^{-3}$ & $9.2110^{-3}$ & $8.2410^{-3}$ & $5.9310^{-3}$ & $5.5310^{-3}$ & $6.6210^{-3}$ \\
$\left\langle\frac{\sigma}{R_{1}}\right\rangle$ & $1.1810^{-2}$ & $9.0310^{-3}$ & $7.8810^{-3}$ & $9.0410^{-3}$ & $9.4310^{-3}$ & $1.1210^{-2}$ \\
$\left\langle\frac{\sigma}{R_{2}}\right\rangle$ & $7.9210^{-3}$ & $1.1210^{-2}$ & $9.9510^{-3}$ & $7.3310^{-3}$ & $6.6610^{-3}$ & $8.0610^{-3}$ \\
$\frac{\operatorname{rms}\left(R_{1}\right)}{\left\langle R_{1}>\right.}$ & $2.0010^{-2}$ & $2.8310^{-2}$ & $2.0610^{-2}$ & $2.7310^{-2}$ & $1.7010^{-2}$ & $1.1210^{-2}$ \\
$\frac{\operatorname{rms}\left(R_{2}\right)}{<R_{2}>}$ & $7.1110^{-3}$ & $1.1210^{-2}$ & $9.8610^{-3}$ & $8.1310^{-3}$ & $6.0210^{-3}$ & $7.5110^{-3}$ \\
$\left\langle\chi^{2}{ }_{1}\right\rangle$ & 3.4099 & 11.4284 & 6.5732 & 9.7096 & 3.5122 & 0.9919 \\
$\left\langle\chi^{2}{ }_{2}\right\rangle$ & 0.9232 & 1.2398 & 1.0428 & 1.2565 & 0.8501 & 0.8553 \\
$\nu$ & 7 & 13 & 17 & 29 & 29 & 37 \\
\hline \hline
\end{tabular}

in about 3 minutes. 4. On 1995 February 8 (Fig. 4) there was a first decline of $0.1 \mathrm{mag}$ during two hours, then a rise and a second decline of 0.06 mag during 40 minutes. 5 . On 1995 February 22 (Fig. 5), the brightness of the blazar was going down slowly. 6. On 1995 February 23 (Fig. 6) no rapid variations appeared.

Table 4 lists the average of relative errors on the fluxes of the blazar, of star 4 and 10 (Row [2], [3] and [4]) and of $R_{1}$ and $R_{2}$ (Row [5] and [6]), the rms values of $R_{1}$ and $R_{2}$ relative to its average (Row [7] and [8]) and the reduced $\chi_{1}^{2}$ and $\chi_{2}^{2}$ of $R_{1}$ and $R_{2}$ (Row [9] and [10]).
From this data, one sees that the variations of OJ 287 are real with a confidence level of 0.9985 (11 April 1994) or larger, except on 23 February 1995 for which no significant variations were observed. Star 5 is too faint to be used as a comparison star for such small variations.

\section{Discussion}

Variations on timescales of minutes, hours and one day were observed in our observations. The apparent efficiency for conversion of accreted matter into energy is $\eta=3.1$ if 

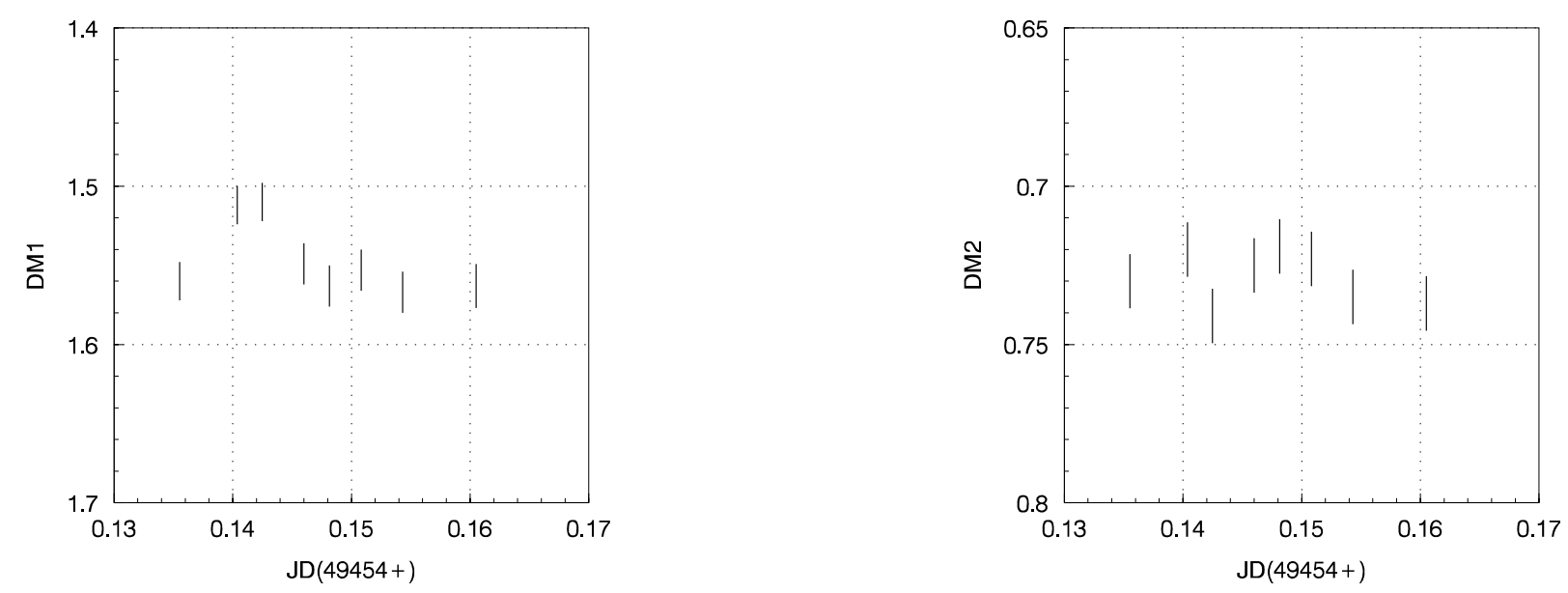

Fig. 1. Light curves of differential magnitude in the $I$ band on 11th April 1994. Dm1 (left) is the differential magnitude between OJ 287 and comparison star 4. Dm2 (right) is the same between comparison stars 10 and 4
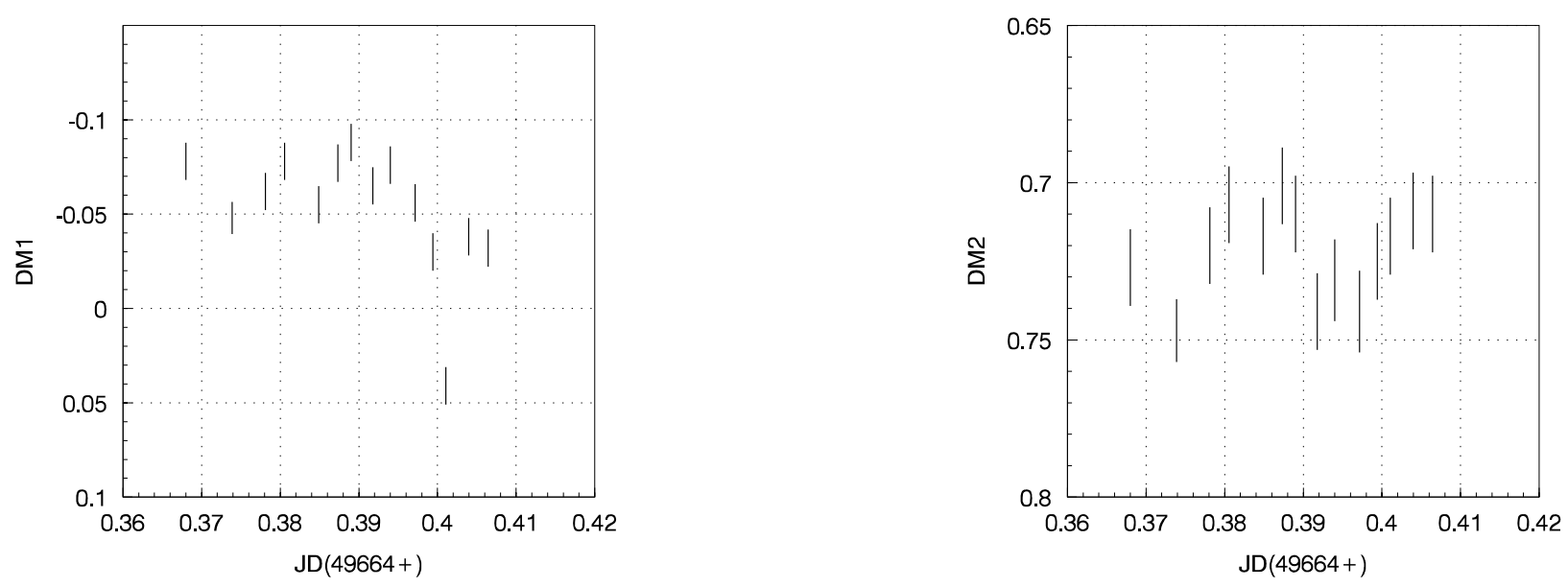

Fig. 2. Light curves of differential magnitude in the $I$ band on 7 th November 1994. Dm1 (left) is the differential magnitude between OJ 287 and comparison star 4. Dm2 (right) is the same between comparison stars 10 and 4

$\Delta m_{I}=0.12$ within $\Delta t=10$ minutes as on 1994 November 7 (Guibin et al. 1998). If $\Delta m_{I}=0.09 \mathrm{mag}$ in $\Delta t=3 \mathrm{~min}$ utes as on 1994 November 8 , the $\eta=5.53$. No matter what the exact value of $\eta$ is, there must be relativistic beaming to explain microvariability, since $\eta$ is always larger than 0.1. The size of the emitting region corresponding to the fastest variation (in a timescale of minutes) is about $510^{13} \mathrm{~cm}$ in the frame of the blazar if a Doppler factor 5 is assumed (Valtaoja et al. 1994). On the basis of the smallest observed periods, the mass of central black hole is less than $510^{7} M_{\odot}$, and the radius of the event horizon of central black hole is less than $10^{13} \mathrm{~cm}$, according to the formula given by Wiita (1985). The size of the emitting re- gion corresponding to the fastest variations is larger than the radius of event horizon of the black hole. Therefore, the rapid variability might result from vortices and magnetic flux tubes in the accretion disk, which facilitate the escape of collimated radiation from deep within the hot disk, producing rapid flux variability (Abramowicz 1992). The variations could also come from shocks within the jet. Shocks in turbulent jets will lead to variations of local emissivity and produce outbursts, due to compression of magnetic field in the shock (Hughes et al. 1985; Königl \& Choudhuri 1985; Jones 1988); fluctuations of injection of particles and acceleration process may give rise to rapid variability. 

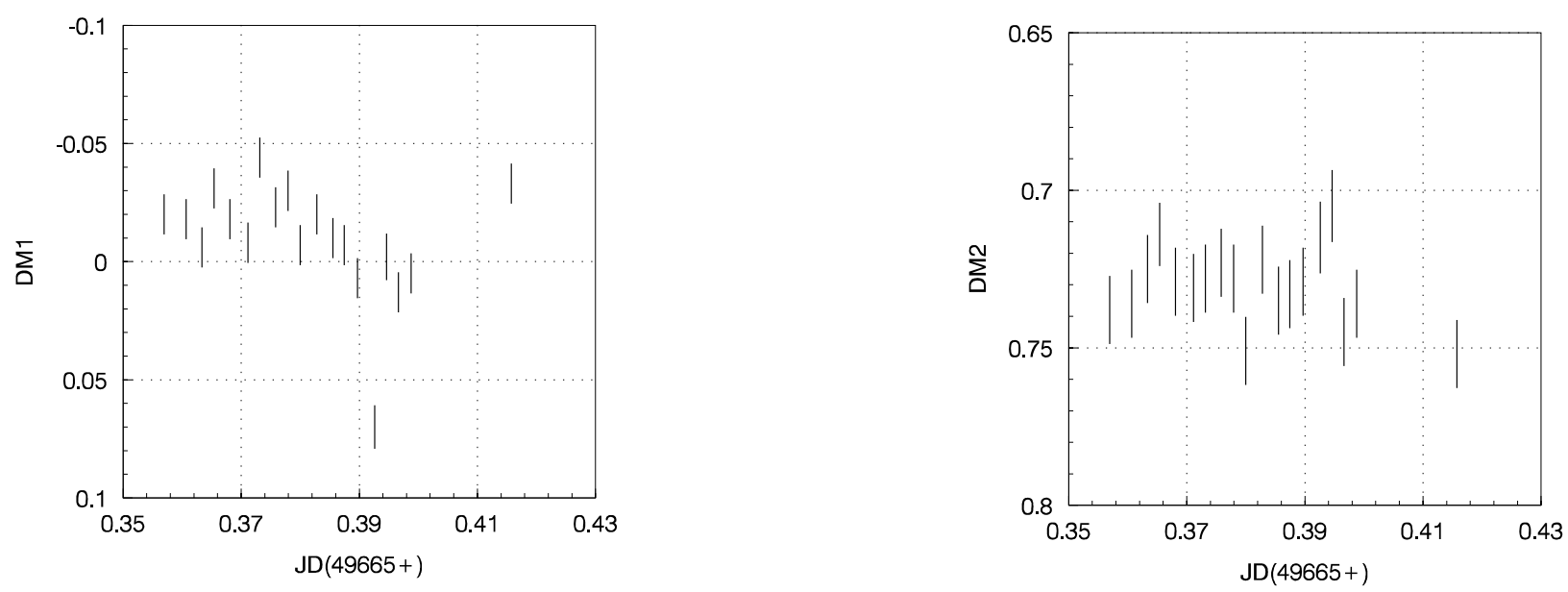

Fig. 3. Light curves of differential magnitude in the $I$ band on 8th November 1994. Dm1 (left) is the differential magnitude between OJ 287 and comparison star 4. Dm2 (right) is the same between comparison stars 10 and 4
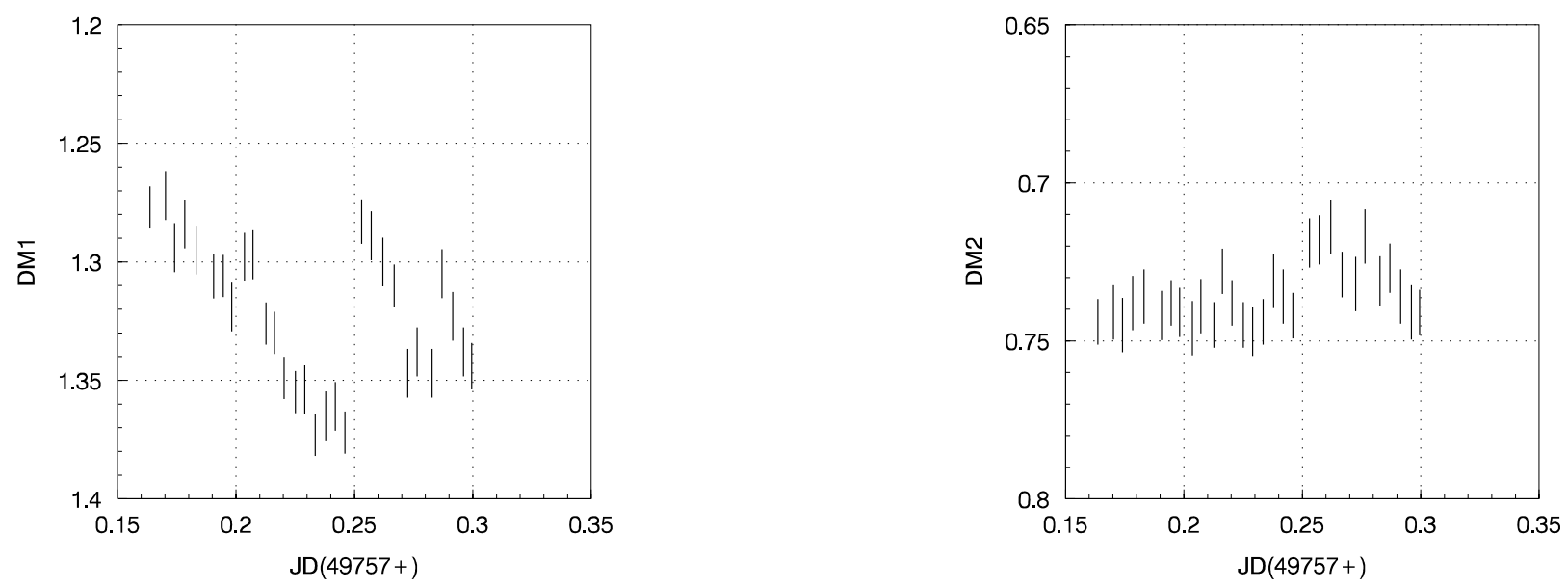

Fig. 4. Light curves of differential magnitude in the $I$ band on 8th February 1995. Dm1 (left) is the differential magnitude between OJ 287 and comparison star 4. Dm2 (right) is the same between comparison stars 10 and 4

\section{Conclusion}

During the observing seasons, OJ 287 was brightening. Its luminosity reached a maximum in November 1995, afterwards, declined and in February 1995 dropped down to nearly the 1994 April level. Variability with timescales of minutes, hours and one day was observed. Our fast photometry shows that there has been variability during the sampling interval in five nights out of six, with rms amplitude of flux variations $1.7 \%-2.8 \%$. The smallest average sampling interval in these nights is 3.4 minutes, during which the rms amplitude was about $2.1 \%$. The largest rms amplitude $(2.8 \%)$ occured within an average sampling interval of 4.7 minutes on 1994 November 7. These rapid variations could result from shocks within turbulent jet or from vortices and magnetic flux tubes on accretion disk.

Acknowledgements. We wish to thank the anonymous referee for his careful reading of the original manuscript and useful suggestions that enabled us to improve the presentation of this work. We also wish to thank the staff of the Optical Union Opening Observatory in the Yunnan Base and of the computer department in the Yunnan Observatory for their help in observations and data processing. This work was partly supported by the astronomical committee of the Chinese Academy of Science and climb project in China.

\section{References}

Abramowicz M.A., et al., 1992, Nat 356, 41 

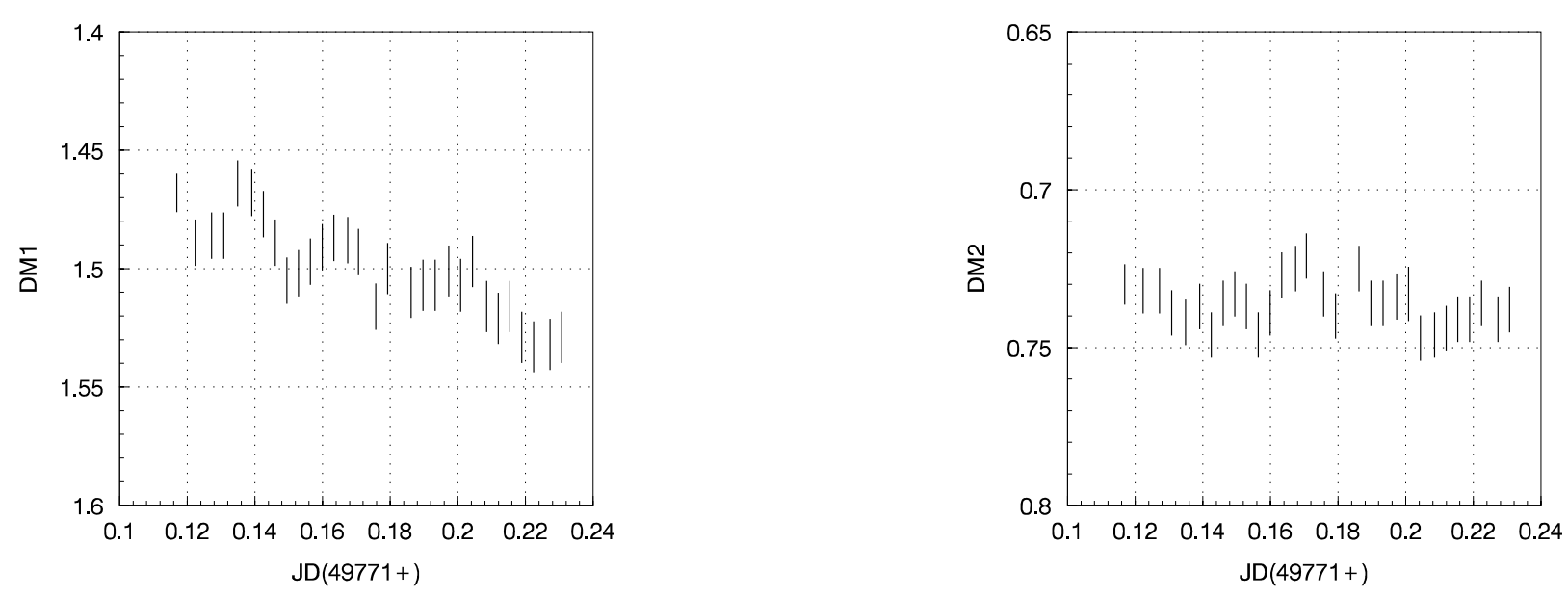

Fig. 5. Light curves of differential magnitude in the $I$ band on 22th February 1995. Dm1 (left) is the differential magnitude between OJ 287 and comparison star 4. Dm2 (right) is the same between comparison stars 10 and 4
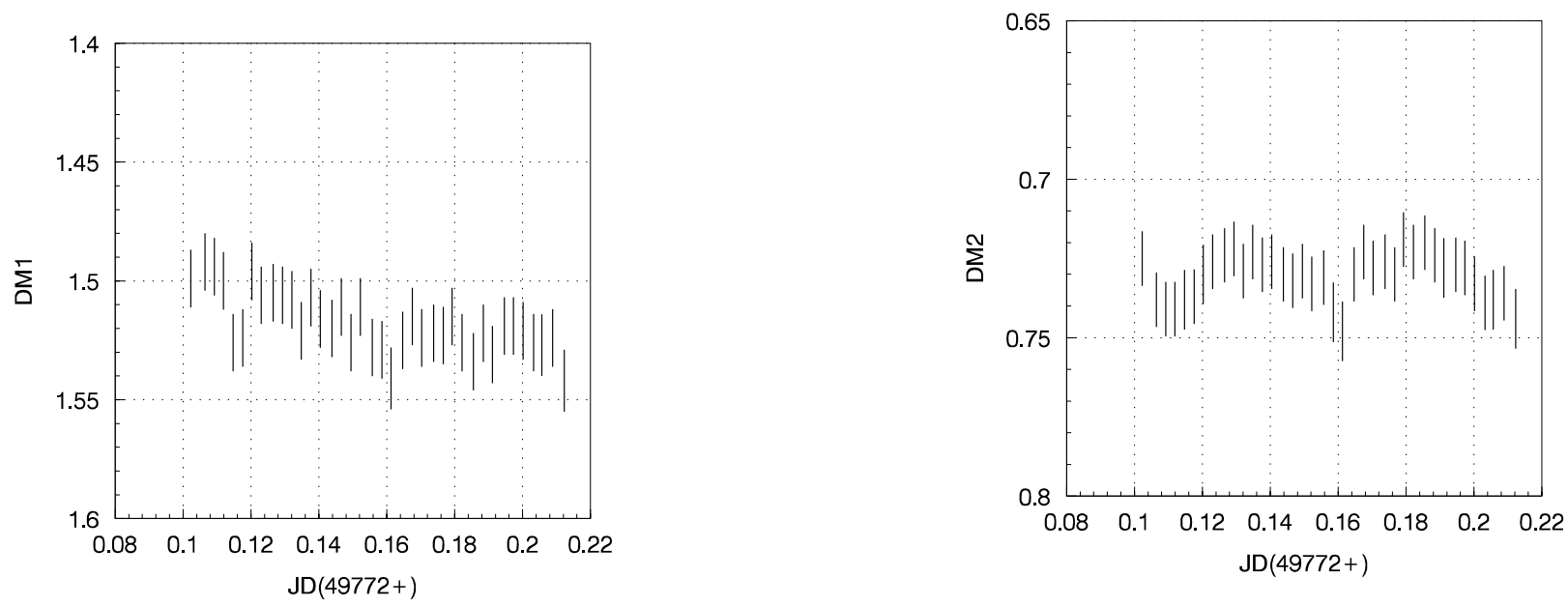

Fig. 6. Light curves of differential magnitude in the $I$ band on 23th February 1995. Dm1 (left) is the differential magnitude between OJ 287 and comparison star 4. Dm2 (right) is the same between comparison stars 10 and 4

Benitez E., Dultzin-Hacyan D., Argaiz D., 1994, Workshop on Intensive Monitoring of OJ 287 Kidger M.R. and Takolo L.O. (eds.), p. 74

Carini M.T., et al., 1992, AJ 104, 15

Carrasco L., Dultyin-Hacyan D., Cruz-Gonzalez I., 1985, Nat 314,146

Dreher W., Roberts D.H., Lehàr J., 1986, Nat 320, 239

De Bruyn A.G., 1988, IAU Symposium 129, the Impact of VLBI on Astrophysics and Geophysics, Reid M.J. and Morgan J.M. (eds.). Dordrecht: Reidel, p. 97

Epstein E.E., et al., 1972, ApJ 178, L51

Frohlich A., Goldsmith S., Weistrop D., 1974, MNRAS 168, 417

Guibin J., et al., 1995, A\&AS 114, 337

Guibin J., et al., 1998, A\&AS 128, 1

Hagen-Thorn V., 1980, A\&ApS 73, 263
Holmes P.A., et al., 1984, MNRAS 211, 497

Hughes P.A., Aller H.D., Aller M.F., 1985, ApJ 298, 301

Jones T.W., 1988, ApJ 332, 678

Kinman T.D., et al., 1974, AJ 79, 349

Königl A., Choudhuri A.R., 1985, ApJ 289, 173

Kinzel W.M., Dickman R.L., Predmore C.R., 1988, Nat 331, 48

Kidger M.R., Takalo L.O., Silanpää A., 1992, A\&A 264, 32

Kidger M.R., Takalo L.O., De Diego J.A., 1994, A\&A 282, 369 Kidger M.R., et al., 1995, A\&AS 113, 431

Lorenzetti D., et al., 1989, ApJS 71, 175

McGimsey B.Q., et al., 1975, AJ 80, 895

Maraschi L., et al., 1986, ApJ 304, 637

Miller H.R., Carini M.F., 1989, In BL Lac Objects, Marachi L., Maccacaro T. and Ulrich U-H. (eds.). Springer verlag, p. 143 
Schild R.E., 1983, PASP 95, 1021

Smith P.S., et al., 1985, AJ 90, 1185

Sillanpää A., et al., 1988, ApJ 325, 628

Sillanpää A., et al., 1992, A\&A 254, L33

Sillanpää A., et al., 1994, Workshop on Intensive Monitoring of OJ 287, Tuorla Observatory reports, Informo No. 174, Kidger M.R. and Takolo L.O. (eds.), p. 7

Sillanpää A., et al., 1996, A\&A 305, L17

Takolo L.O., et al., 1992, AJ 104, 40

Takolo L.O., et al., 1994, A\&AS 104, 115

Turner G.W., Honeycutt R.K., Robertson J.W., 1994 Workshop on Intensive monitoring of OJ 287, Kidger M.R. and Takolo L.O. (eds.), p. 69

Valtaoja E., et al., 1985, Nat 314, 148
Valtaoja E., et al., 1991, AJ 101, 78

Valtaoja E., Terästranta H., Tornikoski M., 1994, Workshop on Intensive Monitoring of OJ 287, Tuorla Observatory Reports, Informo No. 174, Kidger M.R. and Takalo L.O. (eds.), p. 55

Visvanathan N., Elliot J.L., 1973, ApJ 179, 721

Véron P., Véron M.P., 1975 A\&A 39, 281

Wolstencroft R.D., Gilmore G., Williams P.M., 1982, MNRAS 201,479

Wiita P.J., 1985, Phys. Rep. 123, 117

Webb J.R., et al., 1988, AJ 95, 374

Wagner S.J., Witzel A., 1995, ARA\&A 33, 163

Xie G.Z., et al., 1987, A\&AS 67, 17

Zaninetti L., Van Horn H.M., 1988, A\&A 189, 45 